\title{
Virtual Biochemistry - pH effect on enzyme activity
}

Heidrich, D.N., Antônio, R.V., Figueiredo, M.S.R.B., Sugai, J.K., Angotti, J.A.P. ${ }^{1}$,

Departamento de Bioquímica, Centro de Ciências Biológicas,

1 Departamento de Metodologia do Ensino, Centro de Ciências da Educação, Universidade Federal de Santa Catarina, Florianópolis/SC, Brasil.

Protocols of laboratory experiments, followed by teacher's explanation, not always clearly translate to the student the dynamics to be adopted for the implementation of the proposed practice. One of these cases is related to the study of the effect of $\mathrm{pH}$ on enzyme activity. For better help the understanding of the technical procedure, a hypermedia was built based on a protocol adopted at the Department of Biochemistry, UFSC. The hypermedia shows how the effect of variations in $\mathrm{pH}$ can be observed in vitro. Taking as example salivary amylase and the consumption of starch (substrate) by means of iodine staining, a set of $\mathrm{pH}$ buffers was tested to identify the best $\mathrm{pH}$ for this enzyme activity. This hypermedia as introductory tool for such practice was tested on a Nutrition course classroom. Students agree that the hypermedia provided a better understanding of the proposed activities. Teachers also notice a smaller reagents consumption and reduction of the time spent by the students in the achievement of the experiment.

Keywords: biochemical hypermedia, $\mathrm{pH}$ effect, salivary amylase, virtual experiment.

Supported by: CNPq 